\title{
Fabry-Perot Spectroscopy of Extremely Faint Astronomical Sources
}

\author{
F. L. Roesler, R. J. Reynolds and F. Scherb, \\ Department of Physics, University of Wisconsin, Madison, WI 53706, \\ $U S A$
}

\begin{abstract}
Our interests in the general field of astrophysics lie principally in two areas: 1) the study of extremely faint emission lines from the interstellar medium, galactic halo, and intergalactic clouds, and 2) the study of neutral and ionized components of the outer atmospheres of solar system objects, including the earth. These studies require instruments of the highest possible area-8olid angle product, but typically do not require extremely high spatial resolution. This paper highlights our past work in these areas, and discusses new instrumental approaches we are developing.
\end{abstract}

\section{Introduction}

This paper has two main themes. First, it reviews the major contributions of our collaboration of more than twenty years in the application of Fabry-Perot interferometers for 3-D spectroscopic studies of extremely faint, spatially extended astronomical emission line sources. Second, it briefly describes new methods we are developing with the expectation of greatly improved sensitivity for advancing our scientific horizons. We have worked in a broad range of astronomy topics, among which the most notable are the warm ionized component of the interstellar medium (WIM), intergalactic clouds, and solar system studies of the extended atmospheres of comets and planets (including earth).

\section{Source Spectral Characteristics}

There is, of course, a close link between the spectral characteristics of the sources we observe and our instrumental approach. The line profiles and absolute intensities of extremely faint emission lines convey the physical information needed to understand the spatially-extended regions of interest. As a practical matter it must always be assumed when working at extremely low light levels that the spectral region to be studied is continuous and crowded because of spectral features from airglow (often variable and 10 or more times brighter than the desired line) or from astronomical features of typically unknown origin. A resolving power of about $25,000\left(12 \mathrm{~km} \mathrm{~s}^{-1}\right)$ or larger is required to extract dynamical information and to separate background features. 


\section{Instrumentation}

Figure 1 shows the layout of our most often used Fabry-Perot spectrometer configuration. Light is guided into the dual-etalon, $145 \mathrm{~mm}$ clear aperture system from a siderostat or telescope giving appropriate (often unit-) magnification. An aperture $A$ in the focal plane of $L_{1}$ isolates the desired spectral and/or spatial region. The light, recollimated by $L_{3}$, is passed through interference filter $F$ which isolates the spectrally pure region provided by the dual etalon. The flip mirror is used to select a photomultiplier when the instrument is used in the scanning mode, or the CCD camera for the spectrally resolved imaging or multiple annular element detection mode (discussed more fully later). The etalons are coated in our laboratory with $91 \pm 1 \%$ broadband coatings modified from the Trauger (1976) design by Tscharnack (unpublished) for the spectral regions $450-900 \mathrm{~nm}$ or $550-1100 \mathrm{~nm}$. The double etalon system allows us to achieve high spectral purity and contrast at high resolution by extending the free spectral range of a single etalon by a factor of about 10 through careful choice of spacer ratios. When the spacers are of comparable magnitudes, an additional important advantage is achieved because of suppression of the troublesome extended Lorentzian wings of the single-etalon line profile. The improved profile and contrast are very helpful for dynamical studies, and for the clean identification of faint features in the midst of bright background features. The etalons are pressure-tuned and scanned. We do not plan to adopt the widely-used fixed-gap, stabilized, PZT scanned systems for several reasons: Our tuning and mounting method provides great freedom in optimizing and modifying our system for the widest variety of problems. Our system can, by comparison, be trivially and economically extended from single to double-and triple-etalon configurations. Our technology is well developed and well demonstrated to be appropriate for our scientific goals.

A Fabry-Perot observatory using an instrument of this type has been in operation at Wisconsin since 1976. A second, similar instrument has been used for remote observations at other facilities, and as an additional instrument at Wisconsin. For more details on our system see Roesler (1974) and Roesler et al. (1979).

\section{The Warm Ionized Interstellar Medium}

One of the major contributions from our Wisconsin Fabry-Perot observatory has been an extensive series of measurements of the faint galactic glow of $\mathrm{H} \alpha \lambda 6563$, [NII] $\lambda 6584$, and [SII] $\lambda 6717$, extending to high galactic latitudes, that have helped establish the existence of a major component of the interstellar medium called the Warm Ionized Medium, or WIM (Reynolds 1990). This medium is characterized typically by the following properties: $\mathrm{EM} \simeq 2 \mathrm{~cm}-6 p c, n_{e} \simeq$ $0.1 \mathrm{~cm}^{-3}, T \simeq 7000 \mathrm{~K}$, and mass of $\mathrm{H}^{+} \simeq 1 / 3$ the mass of the neutral hydrogen. These measurements have forced a profound rethinking of the composition and structure of the ISM from the way it was viewed when our measurements were begun in the late ' 60 's, and has triggered a search for the source of the ionization (Kulkarni and Heiles 1987; Cox 1989; Dettmar 1992). 




Figure 1. Typical optical schematic for a dual-etalon FabryPerot/CCD spectrometer.

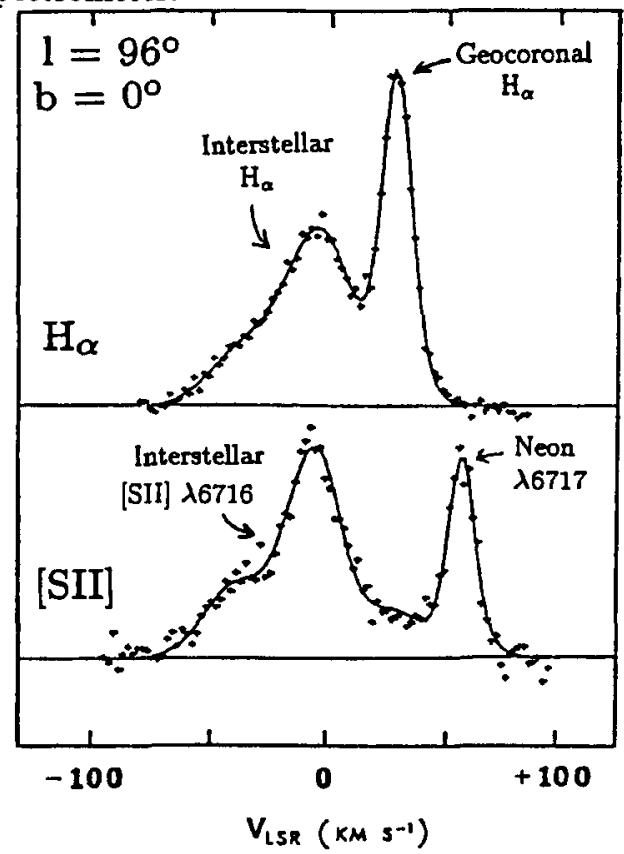

Figure 2. Scans comparing WIM diffuse H $\alpha \lambda 6563$ emission (upper) and [SII] $\lambda 6716$ emission (lower) from the galactic equator $\left(\ell=96^{\circ}, b\right.$ $\left.=0^{\circ}\right)$. The background has been suppressed. 
Fig. 2 shows a pair of scans obtained at the Wisconsin Fabry-Perot observatory from a $3 / 4^{\circ}$ patch of sky on the galactic equator $\left(\ell=96^{\circ}, b=0^{\circ}\right)$. They illustrate typical source spectral features that dictate the need for the instrumental characteristics described earlier. The $\mathrm{H} \alpha$ and [SII] scans both show a strong nearby terrestrial source: the natural geocoronal source of $\mathrm{H} \alpha$ and the Ne line near [SII] from artificial sources. The two spectra clearly show the same gross galactic dynamical effects, but the features are much sharper for the heavier SII ions than for the much lighter hydrogen atoms with faster thermal speeds. Comparison of the line profiles allows separation of large-scale galactic dynamics, local turbulence, and thermal properties of the emitting regions. Comparison of line intensities helps to distinguish WIM emission from traditional HII region emission. For example, the [SII] $/ \mathrm{H} \alpha$ ratio for the WIM is typically 4 times higher than that for traditional HII regions (Reynolds 1985).

From a large number of such $\mathrm{H} \alpha$ scans on a roughly $10^{\circ} \mathrm{grid}$ and extending to high galactic latitudes, a crude, all-sky map has been generated (Reynolds 1990 ). Smaller, $10^{\circ} \times 10^{\circ}$ regions have been mapped dynamically on a $1^{\circ}$ scale (Reynolds 1980, 1987) and illustrate the scientific potential of producing a dynamically resolved, all sky $\mathrm{H} \alpha$ map. We are implementing an all-sky mapping program with a new instrumental technique described below. Data from the mapping done with the existing instrument show interesting relationships between $21 \mathrm{~cm}$ emission from $\mathrm{HI}$ and $\mathrm{H} \alpha$ emission from the WIM. For example, it has been found that roughly $30 \%$ or more of $\mathrm{HII}$ and $10-30 \%$ of the $\mathrm{HI}$ exists in "H $\alpha$-HI clouds" that extend to $1000 \mathrm{pc}$ from the galactic midplane (Reynolds et al. 1994).

\section{H $\alpha$ Emission From Intergalactic Clouds}

Another major contribution from our work was the acquisition of the first evidence of a weak flux of $\mathrm{H} \alpha$ from intergalactic HI clouds. Our initial study (Reynolds et al. 1986) was of the large intergalactic HI cloud discovered in Leo by Schneider et al. (1983). Based on this $21 \mathrm{~cm}$ measurement, we chose two fields on the cloud having different doppler shifts. The two fields were repeatedly scanned over a range including the expected doppler-shifted $\mathrm{H} \alpha$ emission from both fields. The idea was that average airglow features and local galactic features would be the same for both fields, and that the difference spectrum would largely eliminate contaminating features, thus leaving the expected differential signature of the cloud emission. Choosing the two doppler-shifted fields allows one spectrum to serve as the background for the other, thus doubling the observational efficiency.

The left portion of Fig. 3 shows the spectra of the two fields followed, at the bottom, by the difference spectrum. It is clear that the scans of the individual fields are strongly contaminated by line and continum emission of galactic and atmospheric origin (marked $\mathrm{G}$ and $\mathrm{E}$ for [NII] line emission in particular on the figure) that make positive identification of emission from Leo virtually impossible. The difference, however, shows clearly the expected signature. The observations of $\mathrm{H} \alpha$ in Leo were made with one of our instruments installed at the west auxiliary heliostat $(0.8 \mathrm{~m})$ of the McMath-Pierce solar telescope at Kitt Peak. 

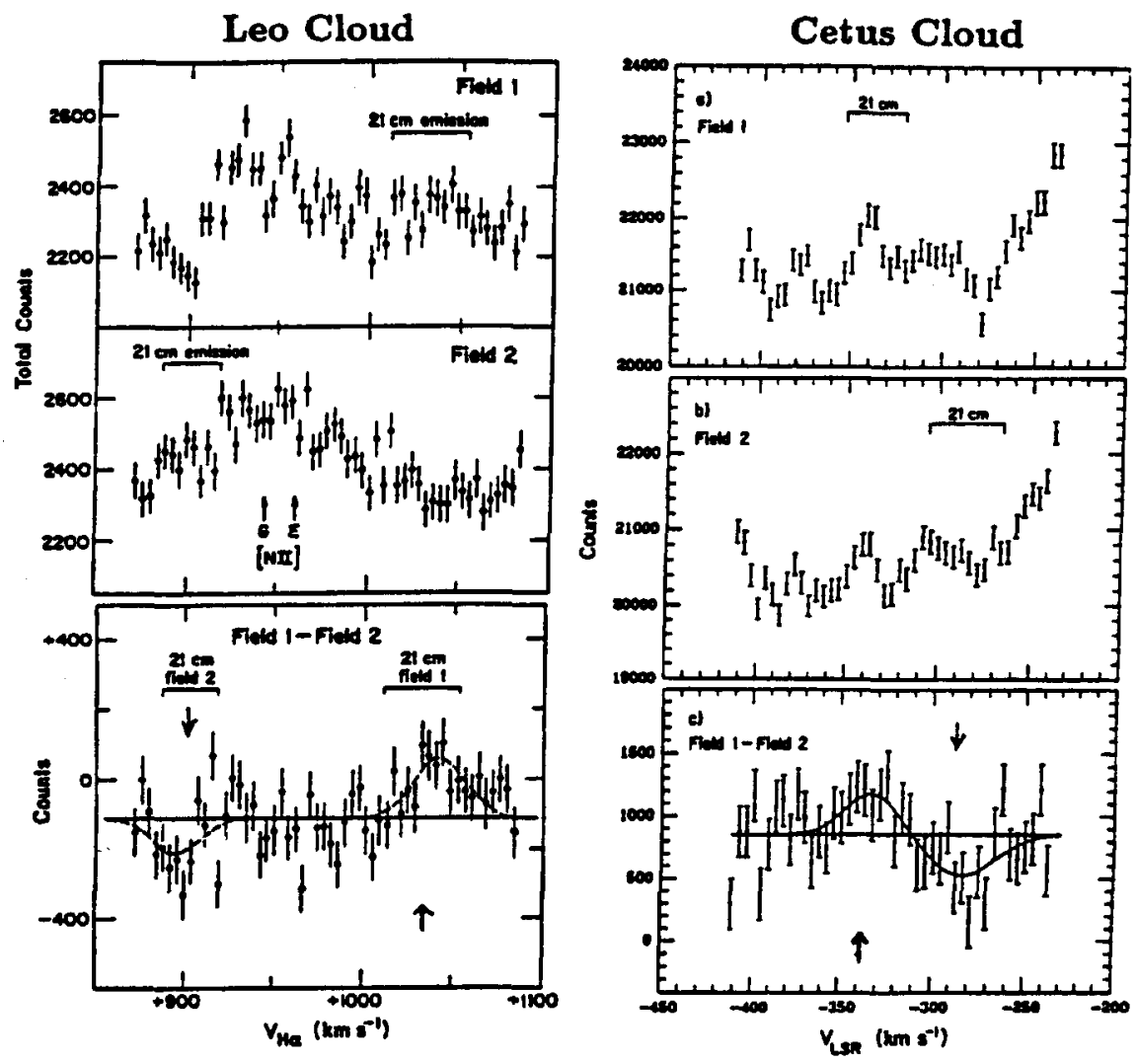

Figure 3. Sequence of scans revealing $\mathrm{H} \alpha$ emission from the intergalactic HI clouds in Leo (left) and in Cetus (right). In each column scans of different fields are shown (upper and middle) with background suppressed. At the bottom, difference spectra show the signatures of cloud emission. 
Another extragalactic cloud nearer to our galaxy has also been observed from our Wisconsin observatory for the purpose of determining its $\mathrm{H} \alpha$ emission rate. The right hand portion of Fig. 3 shows results of observations of a very high velocity cloud in Cetus obtained by Kutyrev and Reynolds (1989) using the same strategy as for the Leo observations. The measured $\mathrm{H} \alpha$ surface brightnes ses are, for the Leo cloud, $0.28 \pm .06 \mathrm{R}$, and for the Cetus cloud $0.08 \pm .02 \mathrm{R}$. (The Rayleigh unit, $R$, is $10^{6} / 4 \pi$ photons $\mathrm{cm}^{-2} \mathrm{~s}^{-1} \mathrm{Sr}^{-1}$.) There is no evidence of ionizing sources within the clouds, and thus an intergalactic Lyman continuum flux $\mathrm{I}_{L C}$ is the probable source of excitation. Assuming the HI clouds are thick to Lyman continuum photons and thin to $\mathrm{H} \alpha$ photons, the expected surface brightness of $\mathrm{H} \alpha$ is $\sim 0.46 \mathrm{I}_{L C}$. Based on our $\mathrm{H} \alpha$ cloud measurements of $\sim$ $0.1 \mathrm{R}$, we obtain $\mathrm{I}_{L C} \sim 2 \times 10^{4} \mathrm{~cm}^{-2} \mathrm{~s}^{-1} \mathrm{Sr}^{-1}$.

\section{The Wisconsin H-Alpha Mapper: WHAM}

We are constructing a second-generation double-Fabry-Perot spectrometer that will be more than ten times as efficient as the instrument we have been using (Reynolds, et al 1990). This instrument will make it possible to provide all-sky, velocity-resolved maps of $\mathrm{H} \alpha$, to extend the observations of diagnostic lines to better understand the excitation of the WIM, and to extend the $\mathrm{H} \alpha$ observations of intergalactic clouds. The sensitivity gain is achieved by application of a CCD imaging detector in the Fabry-Perot annular summing mode, a technique reminiscent of the earliest astronomical applications of the Fabry-Perot. A central region of the Fabry-Perot pattern embracing a large total spectral range of interest is imaged on the CCD, and the field is carved (in software) into nested annular regions of equal spectral width (corresponding to the FabryPerot limit of resolution) and equal solid angle. Thus the central region has radius $r_{1}$ corresponding to the central resolution element, and adjacent annular regions have outer radii $\sqrt{n} r_{1}$ where $n=1,2,3, \ldots N$ for the $N$ elements to be observed. The gain is achieved by the simultaneous observation of $\mathbf{N}$ spectral elements and by the (typically) greater quantum efficiency in the vicinity of $\mathrm{H} \alpha$ for the CCD as compared to the photomultiplier. The overall gain is reduced because of read noise, so the way in which optimum performance is achieved needs to be considered. Basically it is desired to minimize the number of reads without compromising the resolving power.

Figure 4 shows a square array of $P \times P$ pixels of dimension $\epsilon$ (the size of $\epsilon$ may be larger than the basic CCD pixel size due to on-chip binning), and the regions corresponding to the innermost spectral element of radius $r_{1}$ and the outermost spectral element of outer radius $\sqrt{N} r_{1}$. If we make the outer annulus of width $\epsilon$, we make only a small compromise in resolving power for the last few annulii. Thus

$$
\begin{aligned}
& r_{N}-r_{N-1}=\epsilon=r_{1}(\sqrt{N}-\sqrt{N-1}) \\
& \text { and } \\
& 2 r_{N}=2 r_{1} \sqrt{N}=P \epsilon .
\end{aligned}
$$

These lead to a minimum array size of $\mathrm{P} \simeq 4 N$ pixels, and approximately $4 \pi N$ pixels per resolution element 


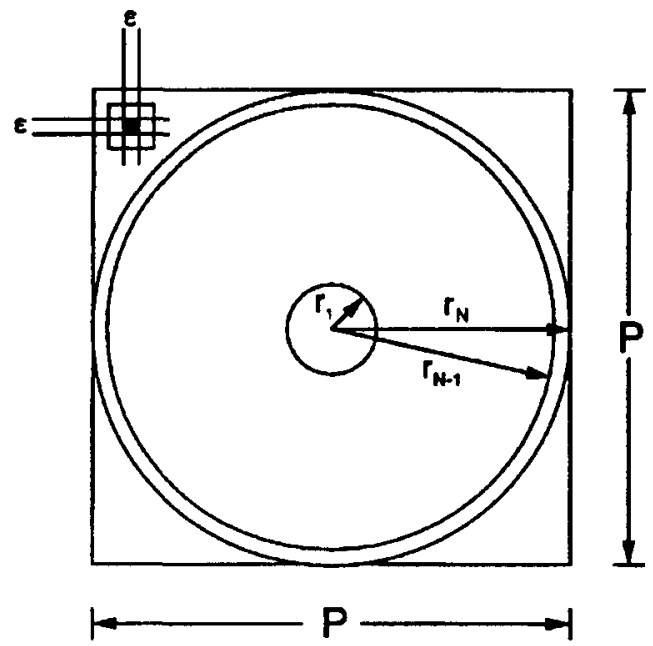

Figure 4. Sketch of the first and Nth Fabry-Perot annuli on a square CCD array for estimating performance in the annular summing mode.

The signal to noise ratio, $\mathrm{S} / \mathrm{N}$, achieved in observing time $T$ may be estimated from the equation

$$
(S / N)=\frac{S}{\sqrt{(S+B+D)+4 \pi N R^{2} / T}} \sqrt{T}
$$

where $S$ and $B$ are signal and background photoelectron rates in an annulus, $D$ is the dark rate in an annulus and $R$ is the rms photoelectron equivalent associated with reading the charge in each (binned) pixel. The observing time required to achieve a particular $\mathrm{S} / \mathrm{N}$ depends in a straightforward way on source intensity, line profile, background intensity, dark rate, instrumental resolving power, étendue, efficiency, read noise, quantum efficiency, CCD setup, and spectral range, but the large number of variables makes a general treatment lengthy. It is evident that for the measurement of very faint sources in the presence of background, the long observing times required to achieve a useful $\mathrm{S} / \mathrm{N}$ tend to diminish the effect of read noise. A comprehensive treatment of the Fabry-Perot annular summing technique is in preparation by Coakley et al. (1994).

The specifications for the WHAM instrument are a $1^{\circ}$ field on the sky, 10 $\mathrm{km} \mathrm{s}^{-1}$ velocity resolution, $200 \mathrm{~km} \mathrm{~s}^{-1}$ range, and $20 \sigma$ detection of one-Rayleigh components ( $E M=2 \mathrm{~cm}^{-6} \mathrm{pc}$ ). The CCD camera is expected to have a $\mathrm{QE}$ of 0.78 at $\mathrm{H} \alpha$ and a read noise of \pm 3 electrons.

The WHAM instrument is basically the same as shown in Fig. 1, but to assure reasonable averaging over the $1^{\circ}$ spatial resolution of the proposed survey, the Fabry-Perot fringe plane will be imaged near the siderostat mirror feeding the system, and the sky will be imaged near the Fabry-Perot. The $200 \mathrm{~km} \mathrm{~s}^{-1}$ range on the chip requires a $4.2^{\circ}$ cone angle through the Fabry-Perot, and thus a $60 \mathrm{~cm}$ objective lens will be used just behind the flat siderostat mirrors to 
reduce the beam diameter to $1^{\circ}$ on the sky. For comparison with our present instrument we estimate that $20 \sigma$ measurements of $1 \mathrm{R}$ lines, which presently take $30 \mathrm{~min}$, will take only 40 seconds with the new instrument, and for $10 \sigma$ measurements of the $\sim 0.1 \mathrm{R}$ lines associated with inter-galactic clouds, the new instrument will require 20 min compared to the current requirement of 50 hours.

We expect to test the new instrument for a year at Wisconsin beginning in early 1995, and begin a 2-year survey at Kitt Peak, Arizona in 1996. A smaller double Fabry-Perot instrument based on concepts identical to those of WHAM is being prepared for use on larger conventional telescopes. Its optics can be modified for operation in the conventional FP/CCD imaging and data cube mode, or in the annular summing mode.

\section{The Hydrogen Geocorona}

In the outermost region of the earth's atmosphere (geocorona), which extends from the exobase at about $400 \mathrm{~km}$ to its merger with the interplanetary medium, the dominant neutral species is hydrogen. It is excited by solar Lyman radiation, and the resulting Balmer-alpha radiation provides a means of studying the excitation, dynamics, hydrogen distribution and temperature in this region of space. Surprisingly, exospheric hydrogen is expected to be sensitive to the buildup of anthropogenic methane in the troposphere through a chemical cycle in which methane augments the delivery of hydrogen into the exosphere (Roble and Dickinson 1989). Our conventional studies extending over the past 14 years have been summarized by Nossal et al. (1994). Our most recent measurements address issues of dynamics and excitation. For these studies we need to determine accurate profiles of lines in the 1 - 5 Rayleigh range at a resolving power of 80,000 . Our application of the FP/CCD annular summing technique for this program has brought 10 to 20 -fold observing time gains. This has allowed us to make fundamental advances in geocoronal studies (Nossal 1994), and in addition to thoroughly validate the principles on which WHAM is based (Coakley et al. 1994).

For data acquisition, the dual etalon is pressure-tuned so that the ring corresponding to the geocoronal line is centered within the 10 spectral element range needed to determine the profile (Fig. 5). The data shown in Fig. 5 were obtained in a $300 \mathrm{~s}$ integration when the line intensity was about $5 \mathrm{R}$. The CCD image resolution was about three times finer than the optimum for WHAM discussed above, but nevertheless a 10-fold gain was achieved in observing time compared to the scanning technique previously used. The pixels within each spectral element are sub-divided into several radial samples (at least two per spectral element are required for fitting), which explains the large number of data points shown in the figure.

The instrumental profile was determined by tuning to a nearby Th hollow cathode line and imaging it to determine its annular sum profile. The data are corrected for pixel-to-pixel response variations and instrumental vignetting by obtaining white light flat fields. We have achieved our best results using a uniformly illuminated white reflecting diffuser placed outside the entire optical train so that it illuminates the system as nearly as possible in the same manner as the source being observed. The off-center appearance of the ring in Fig. 5 

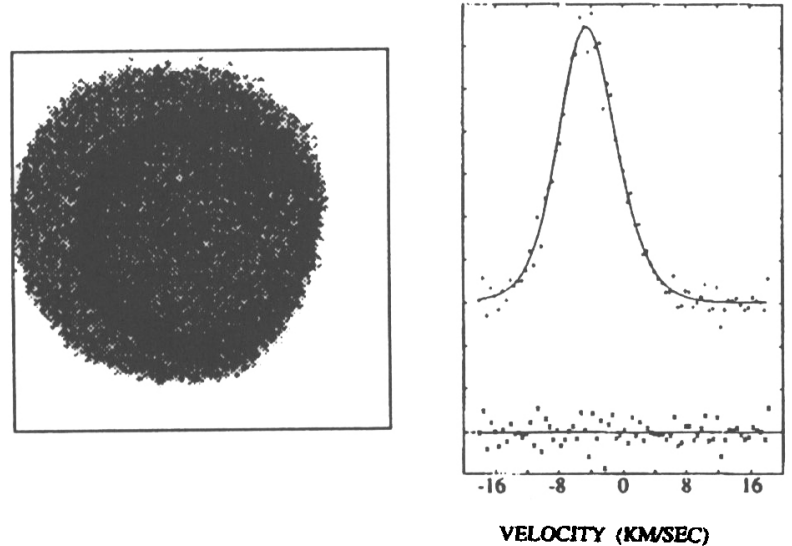

Figure 5. Fabry-Perot ring image of geocoronal $\mathrm{H} \alpha$ emission recorded by a CCD (left) and the line profile (right) derived by annular summing.

results from blanking the pixels in a region where we felt the flat field might be inaccurate. Up to the present time we have relied on absolute intensity determinations using a second scanning instrument to compare the total emission from the direction being observed to previously calibrated standard emission nebulae. We have made progress in developing a calibrated, large area emission line source for directly calibrating the FP/CCD annular summing system.

\section{Solar System Astronomy}

We have chosen to represent our work in this area with a unique set of very high spectral resolution measurements of emission lines from the coma of Comet Halley. Understanding the photochemical kinetics and dynamics of molecules within the coma is an important goal of cometary physics; however the relatively slow speed of cometary molecules leads to very narrow optical emission lines. Although radio emission lines from the coma have yielded much valuable information in this area, the possibilities for using optical emission line profiles have not been fully exploited.

As part of our Comet Halley observing program, we obtained a set of highspectral-resolution measurements on [OI] $\lambda 6300$ and $\mathrm{NH}_{2} \lambda 6298.62$ emissions from the coma in January and May 1986. The successful interpretation of these observations using advanced coma gas dynamic and photochemical models (Combi and Smyth 1988a,b; Combi, Bos and Smyth 1993) provides an important new means for studying the details of coma gas dynamics (Smyth et al. 1994). The [OI] 6300 line profiles were particularly important for studying contrasting cometary outflow conditions. The Halley heliocentric distance in January was small $(\sim 0.78 \mathrm{AU})$ and near perihelion $(0.59 \mathrm{AU})$ so that the $\mathrm{H}_{2} \mathrm{O}$ production rate was near its maximum value, while the heliocentric distance in May was 


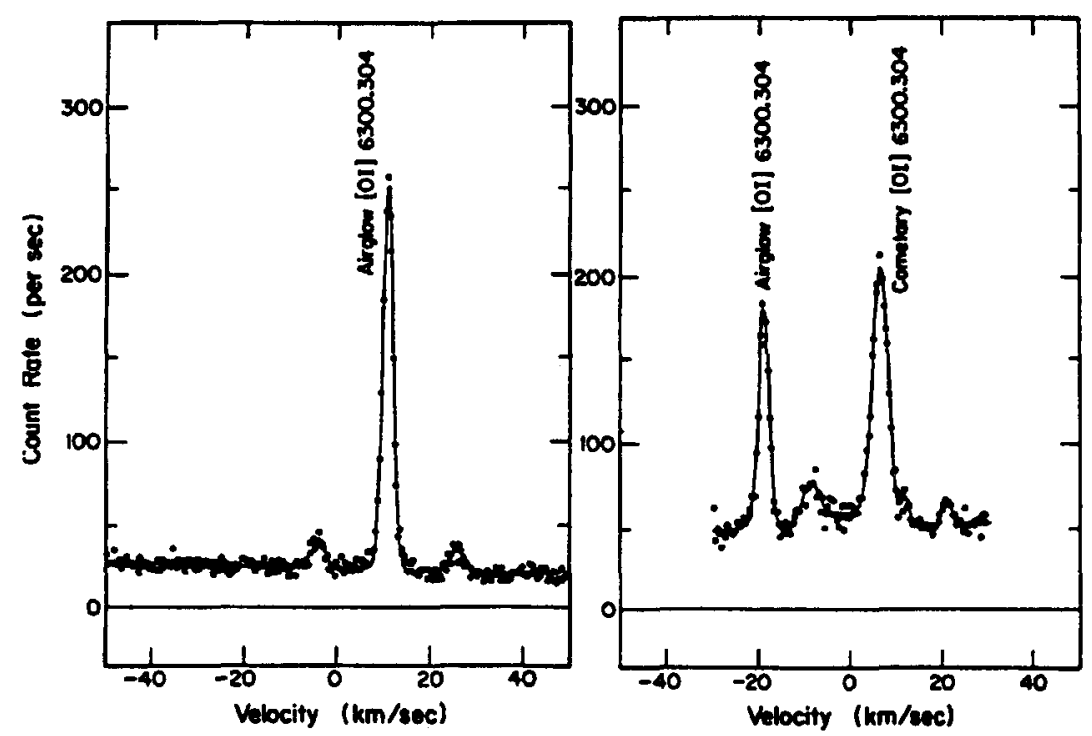

Figure 6. Very high resolution [OI]6300 spectra from the head of Comet Halley (right) and airglow background away from the comet (left). The velocity scale is relative.

much larger ( $1.68 \mathrm{AU}$ ) so that the $\mathrm{H}_{2} \mathrm{O}$ production rate was smaller by approximately an order of magnitude.

For our Halley observations, one of our large dual-etalon, pressure-scanned Fabry-Perot spectrometers (Fig. 1) was installed at the McMath-Pierce solar telescope on Kitt Peak. The instrument was used for a variety of observations, most of which were carried out at a medium spectral resolving power of 30,000 (Reynolds et al. 1986; Magee-Sauer et al. 1988, 1989, 1990; Scherb et al. 1990; Smyth et al. 1993). The conversion of the spectrometer to the highresolution mode $(\lambda / \Delta \lambda=190,000)$ for the cometary line profile studies was effected by replacing one medium-resolution etalon with a high-resolution etalon. The general layout of the instrument and modifications are discussed in Scherb et al. (1986) \& Roesler et al. (1986).

Figure 6 shows a high-resolution [OI] $\lambda 6300$ scan, obtained on 16 January 1986 with the field of view centered on the comet head, and an airglow background scan. The airglow and comet [OI] $\lambda 6300$ emission lines are the prominent features. Other apparent emission features are the combined ghosts from the airglow and comet. The comet emission line is clearly wider than the airglow line.

For Comet Halley, these optical measurements had an additional importance, since they provided water production rates during a critical pre-perihelion period when these rates were near their maximum values and when few measurements, with the exception of radio $\mathrm{OH}$ emission observations, were made. The radio $\mathrm{OH}$ measurements are difficult to interpret because of uncertainties in 
corrections for collisional quenching at high water productions rates (BockeléeMorvan et al. 1990, Gérard 1990, Schloerb 1988). Absolute intensity calibration for our observations in January 1986 allowed the production rate for the $\mathrm{H}_{2} \mathrm{O}$ parent molecules to be calculated (Smyth et al. 1994). The large production rates of $\sim 2.8 \times 10^{30}$ molecules $\mathrm{s}^{-1}$ on January 16 and 17 establish that the maximum water production rate for Comet Halley occurred pre-perihelion in January.

\section{SHS: An Instrumental Technique Under Development}

We are also developing an unusual Fourier spectroscopic technique for faintsource spectroscopy, called Spatial Heterodyne Spectroscopy (SHS), that is based on an unscanned, field-widened Michelson interferometer. Our initial motivation was to bring the advantages of interference spectroscopy to the far ultraviolet spectral region where it appeared that practical problems with materials, surfaces, and coatings might prevent efficient Fabry-Perot operation. We now believe that there are potential applications for SHS at all wavelengths. There is not space here to describe this instrument, and moreover, it falls outside the scope of a Fabry-Perot paper. The brief report on our SHS by Harlander in these proceedings will be helpful, and the paper by Harlander et al. (1993) will give the reader a good introduction to the technique and its history.

Acknowledgments. The work reported in this paper would not have been possible without steady support from the National Science Foundation, the National Aeronautic and Space Administration, and the University of Wisconsin, nor without the interest, encouragement, and dedicated efforts of many, many students and colleagues. We gratefully acknowledge these contributions.

\section{References}

Bockelée-Morvan, D., Crovisiér, J. and Gerard, E. 1990, Astron. and Astrophys. 238, 382.

Coakley, M.M., Roesler, F.L., Reynolds, R.J. and Nossal, S. 1994 in preparation. Combi, M.R., and Smyth, W.H. 1988a, Ap. J. 327, 1026.

Combi, M.R., and Smyth, W.H. 1988b, Ap. J. 327, 1044.

Combi, M.R., Bos, B.J., and Smyth, W.H. 1993, Ap. J. 404, 668.

Cox, D.P. 1989 in IAU Colloquium No. 120, Structure and Dynamics of the Interstellar Medium, eds G. Tenorio-Tagle, M. Moles and J. Melnick (New York: Springer) p.500.

Dettmar, R.J. 1992, Fundamentals of Cosmic Physics 15, 143.

Gerard, E. 1990, Astron. and Astrophys. 230, 489.

Harlander, J., Reynolds, R.J. and Roesler, F.L. 1993, Ap. J. 396, 730.

Kulkarni, S.R. and Heiles, C. 1987 in Interstellar Processes, eds. R.J. Hollenbach and H.A. Thronson, Jr. (Dordrecht: Reidel) p.87.

Kutyrev, A. and Reynolds, R.J. 1989, Ap. J. 344, L9.

Magee-Sauer, K., Roesler, F.L., Scherb, F., Harlander, J., and Oliversen, R.J. 1988, Icarus 76, 89. 
Magee-Sauer, K., Scherb, F., Roesler, F.L., and Harlander, J. 1989, Icarus 82, 50.

Magee-Sauer, K., Scherb, F., Roesler, F.L., and Harlander, J. 1990, Icarus 84, 154.

Nossal, S., Reynolds, R.J., Roesler, F.L., Scherb, F., and Harlander, J. 1993, JGR 98, 3669.

Nossal, S. 1994, PhD Thesis, Dept. of Physics, University of Wisconsin, Madison, WI.

Reynolds, R.J. 1980, Ap. J. 236, 153.

Reynolds, R.J. 1985, Ap. J. 294, 256.

Reynolds, R.J. 1987, Ap. J. 323, 118.

Reynolds, R.J. 1990, in IAU Symposium No. 139, The Galactic and Extragalactic Background Radiation, ed. S. Bowyer and C. Leinert (Dordrecht: Kluwer) p.157.

Reynolds, R.J., Magee, K., Roesler, F.L., Scherb, F. and Harlander, J. 1986, Ap. J. 309, L9.

Reynolds, R.J., Roesler, F.L., Scherb, R. and Harlander, J. 1990, in Astronomical Telescopes and Instrumentation for the 21st Century, SPIE 1235, 610.

Reynolds, R.J., Tufte, S., Kung, D., McCullough, P. and Heiles, C. 1994, in preparation.

Roble, R.G., and Dickenson, R.E. 1989, Geophys. Res. Lett. 16, 1441.

Roesler, F.L. 1974 in Methods of Experimental Physics 12, part A: Optical and Infrared, ed. N. P. Carleton, p.531. (Academic Press, New York.)

Roesler, F.L., Reynolds, R.J., Scherb, F., Ogden, P.M. 1979 in 4th Colloquium of the Trieste Observatory: High resolution Spectroscopy, ed M. Hack, p.600 (Osservatorio Astronomico di Trieste, Trieste).

Roesler, F.L., Scherb, F., Magee, K., Harlander, J., Reynolds, R.J., Yelle, R.V., Broadfoot, A.L., and Oliversen, R.J. 1986, Adv. Space Res. 5, 279.

Scherb, F., Roesler, F.L., Magee, K., Harlander, J., and Reynolds, R.J. 1986, Adv. Space Res. 5, 275.

Scherb, F., Magee-Sauer, K., Roesler, F.L., and Harlander, J. 1990, Icarus 86, 172.

Schloerb, F.P. 1988, Ap. J. 332, 524.

Schneider, S.E., Helou, G., Salpeter, E.E. and Terzian, Y. 1983 Ap. J. 273, L1.

Smyth, W.H., Marconi, M.L., Scherb, F., and Roesler, F.L. 1993, Ap. J. 413, 756.

Smyth, W.H., Combi, M.R., Roesler, F.L., and Scherb, F. 1994, submitted to Ap. J..

Trauger, J.T. 1976, Appl. Opt. 16, 2998. 